\title{
The impact of the new Earth gravity mod- els on the measurement of the Lense-Thirring effect with a new satellite
}

\author{
Lorenzo Iorio \\ Dipartimento Interateneo di Fisica dell' Università di Bari \\ Via Amendola 173, 70126 \\ Bari, Italy \\ e-mail: lorenzo.iorio@libero.it
}

\begin{abstract}
In this paper we investigate the opportunities offered by the new Earth gravity models from the dedicated CHAMP and, especially, GRACE missions to the project of measuring the general relativistic Lense-Thirring effect with a new Earth's artificial satellite. It turns out that it would be possible to abandon the stringent, and expensive, requirements on the orbital geometry of the originally prosed LARES mission (same semimajor axis $a=12270 \mathrm{~km}$ of the existing LAGEOS and inclination $i=70 \mathrm{deg}$ ) by inserting the new spacecraft in a relatively low, and cheaper, orbit $(a=7500-8000 \mathrm{~km}, i \sim 70 \mathrm{deg})$ and suitably combining its node $\Omega$ with those of LAGEOS and LAGEOS II in order to cancel out the first even zonal harmonic coefficients of the multipolar expansion of the terrestrial gravitational potential $J_{2}$, $J_{4}$ along with their temporal variations. The total systematic error due to the mismodelling in the remaining even zonal harmonics would amount to $\sim 1 \%$ and would be insensitive to departures of the inclination from the originally proposed value of many degrees. No semisecular long-period perturbations would be introduced because the period of the node, which is also the period of the solar $K_{1}$ tidal perturbation, would amount to $\sim 10^{2}$ days. Since the coefficient of the node of the new satellite would be smaller than 0.1 for such low altitudes, the impact of the non-gravitational perturbations of it on the proposed combination would be negligible. Then, a particular financial and technological effort for suitably building the satellite in order to minimize the non-conservative accelerations would be unnecessary.
\end{abstract}




\section{Contents}

1 Introduction 3

1.1 The performed tests with the existing LAGEOS and LAGEOS II satellites 3

1.2 The proposed LAGEOS III/LARES/WEBER-SAT satellite . 5

1.3 Aim of the paper . . . . . . . . . . . . 5

2 What can be done with only one new, passive satellite of LAGEOS type? 7

2.1 The originally proposed LAGEOS-LARES scenario: the sum of the nodes 7

2.1.1 Conclusions . . . . . . . . . . . 8

2.2 The LARES-only scenario . . . . . . . . . . . . . . . . . 8

2.2.1 The nearly-polar orbit . . . . . . . . . . . . . . 11

2.2.2 The non-polar scenarid . . . . . . . . . . . . 11

2.2 .3 Conclusions . . . . . . . . . . . . . . 11

2.3 A high-altitude LARES scenarid . . . . . . . . . . . 14

2.4 The linear combination scenario with LAGEOS and LAGEOS II 14

3 What can be done with two new spacecraft? 20

\begin{tabular}{|ll}
4 Conclusions & 21
\end{tabular} 


\section{Introduction}

The general relativistic gravitomagnetic Lense-Thirring effect on the orbit of a test particle (Lense and Thirring 1918) consists of secular precessions of the longitude of the ascending node $\Omega$ and the argument of the pericentre $\omega$ of the orbit of a test particle in geodesic motion around a central spinning mass with proper angular momentum $J$

$$
\dot{\Omega}_{\mathrm{LT}}=\frac{2 G J}{c^{2} a^{3}\left(1-e^{2}\right)^{3 / 2}}, \dot{\omega}_{\mathrm{LT}}=-\frac{6 G J \cos i}{c^{2} a^{3}\left(1-e^{2}\right)^{3 / 2}},
$$

where $G$ is the Newtonian gravitational constant and $a, i, e$ are the semimajor axis, the inclination to the equatorial plane of the central mass and the eccentricity, respectively, of the test particle's orbit.

Up to now, a clear and undisputable direct test of such a general relativistic prediction, which may have important consequences in many astrophysical scenarios involving, e.g., accreting disks around black holes (Stella et al. 2003), is not yet available. However, according to K. Nordtvedt gravitomagnetism would have already been indirectly tested from the radial motion of the LAGEOS Earth satellite (Nordtvedt 1988) and from the highprecision reconstruction of the lunar orbit with the Lunar Laser Ranging (LLR) technique (Nordtvedt 2003).

In April 2004 the GP-B mission (Everitt et al. 2001) has been launched. Its aim is the measurement of another gravitomagnetic effect, i.e. the precession of the spins (Schiff 1960) of four superconducting gyroscopes carried onboard with a claimed accuracy of $1 \%$ or better.

\subsection{The performed tests with the existing LAGEOS and LA- GEOS II satellites}

Up to now, the only performed tests aimed at the explicit and direct detection of such a post-Newtonian effect in the gravitational field of the Earth have been performed by Ciufolini and coworkers by analyzing the data from the existing Satellite Laser Ranging (SLR) LAGEOS and LAGEOS II satellites (Ciufolini et al. 1998, Ciufolini and Pavlis 2004). The claimed total accuracy is $20-30 \%$ (1-sigma) and, for the most recent test, $5-10 \%$ (1-3 sigma). However, the evaluation of the total error budget, including the impact of various sources of systematic errors induced by many gravitational (static and time-dependent parts of the Earth's gravitational potential; Iorio 2001, 2003d) and non-gravitational (direct solar radiation pressure, Earth's 
albedo, Earth's infrared radiation, asymmetric reflectivity, solar YarkovskySchach and Earth's Yarkovsky-Rubincam thermal thrusts; Lucchesi 2001, 2002, 2003, 2004, Lucchesi e al. 2004) perturbations has been critically discussed by a number of authors (Ries et al. 2003, Vespe and Rutigliano 2004, Iorio 2005a) who pointed out that such evaluations are to be considered optimistic.

This is particularly true for the tests involving the nodes of LAGEOS and LAGEOS II and the perigee of LAGEOS II (Ciufolini et al. 1998) based on a suitable linear combination of them proposed in (Ciufolini 1996). Indeed, in that case the total error might be close to $100 \%$ (1-sigma) mainly due to the impact of the non-gravitational perturbations, which severely affect the perigee of the LAGEOS-like satellites, and of the mismodelling in the static part of the even zonal harmonic coefficients $J_{\ell}$ of the multipolar expansion of the terrestrial gravitational potential. Indeed, according to the pre-CHAMP/GRACE era EGM96 Earth gravity model (Lemoine et al. 1998) adopted in that analysis, the systematic error due to the even zonal harmonics would be $\sim 80 \%$ (Ries et al. 2003, Vespe and Rutigliano 2004, Iorio 2003d, 2005a) at 1-sigma.

In the case of the more precise test involving only the nodes of the LAGEOS satellites (Ciufolini and Pavlis 2004), based on a suitable linear combination of them proposed in (Iorio and Morea 2004) and the EIGENGRACE02S Earth gravity model (Reigber et al. 2005), the impact of the secular variations $\dot{J}_{\ell}$ of the low-degree even zonal harmonics of the geopotential may be a further biasing effect. According to (Iorio 2005a), the total error could be $\sim 15-45 \%$ at 1-3 sigma over the 11-year time span of the performed analysis.

A possible way to solve such problems has recently been proposed in (Iorio and Doornbos 2005). It consists in suitably combining the nodes of the existing geodetic LAGEOS, LAGEOS II and Ajisai satellites and of the altimeter Jason-1 satellite in order to cancel out $J_{2}, J_{4}, J_{6}$ along with their secular variations. The systematic error due to the remaining even zonal harmonics is $<2 \%$ according to the recently released EIGEN-CG01C Earth gravity model (Reigber et al. 2004) which combines data from CHAMP, GRACE and terrestrial gravimetry and altimetry measurements. However, the reduction of the data of Ajisai and, especially, Jason-1 in a truly dynamical way to a level comparable to that of the LAGEOS satellites would not be a trivial task, mainly due to the action of the non-gravitational perturbations. However, it must be pointed out that the coefficients with which Ajisai and Jason- 1 enter this combination are of the order of $10^{-3}-10^{-2}$ and no very long period sinusoidal perturbations are introduced. This feature is 
Table 1: Orbital parameters of the existing LAGEOS and LAGEOS II and of the proposed LARES and their Lense-Thirring node precessions in milliarcseconds per year $\left(\right.$ mas $\left.\mathrm{yr}^{-1}\right)$.

\begin{tabular}{lllll}
\hline Satellite & $a(\mathrm{~km})$ & $e$ & $i(\mathrm{deg})$ & $\dot{\Omega}_{\mathrm{LT}}\left(\mathrm{mas} \mathrm{yr}^{-1}\right)$ \\
\hline LAGEOS & 12270 & 0.0045 & 110 & 31 \\
LAGEOS II & 12163 & 0.0135 & 52.64 & 31.5 \\
LARES & 12270 & 0.04 & 70 & 31 \\
\hline
\end{tabular}

very important because it would, thus, be possible to fit and remove them from the signal over a not too long observational time span.

\subsection{The proposed LAGEOS III/LARES/WEBER-SAT satel- lite}

In 1986 (Ciufolini 1986), Ciufolini proposed to orbit a LAGEOS-like satelliteknown also as LAGEOS III (Tapley and Ciufolini 1989), LARES (Ciufolini 1998), WEBER-SAT (Ciufolini et al. 2004)-with the same orbital parameters of LAGEOS, apart from the inclination which should have an offset of $180 \mathrm{deg}$, in order to cancel out the impact of all the even zonal harmonics of the geopotential, at least in principle. Indeed, while the nodal LenseThirring precessions are independent of the inclination of the orbital planes, the classical nodal precessions are proportional to $\cos i$, so that, by using the sum of the nodes of LAGEOS and its twin, the gravitomagnetic rates would sum up while the aliasing classical rates would subtracted.

In Table 1 we quote the orbital parameters of the existing LAGEOS satellites and of the proposed LARES.

\subsection{Aim of the paper}

In this paper we wish to investigate the impact that the new Earth gravity models from the dedicated CHAMP (http://www.gfz-potsdam.de/pb1/op/champ/index_CHAMP.html and GRACE (http://www.gfz-potsdam.de/pb1/op/grace/index_GRACE.html) missions might have on a space mission involving the launch of only one new spacecraft (Section 2) or two new spacecraft (Section 3) aimed at the measurement of the Lense-Thirring effect. In Table 2] we show the calibrated sigmas of the even zonal harmonic coefficients of the geopotential of the pre-CHAMP/GRACE EGM96 Earth gravity model and of some of the most recent GRACE/CHAMP-based solutions EIGEN-GRACE02S and EIGEN-CG01C, released by the GeoForschungsZentrum (GFZ), Potsdam, 
and GGM02S (http://www.csr.utexas.edu/grace/gravity/), released by the Centre for Space research (CSR), Austin. We will show that, with only

Table 2: Errors in the even zonal normalized Stokes coefficients $\sigma_{\bar{C}_{\ell 0}}$ for various Earth gravity models up to degree $\ell=20$. It is important to note that, while EGM96 is based on a multidecadal time span, the CHAMP and GRACE models presented here have been obtained from observational intervals of a few months: 111 days from GRACE for EIGEN-GRACE02S, 860 days from CHAMP and 200 days from GRACE (plus terrestrial gravimetry and altimetry data) for EIGEN-CG01C, 363 days from GRACE for GGM02S.

\begin{tabular}{lllll}
\hline$\ell$ & EGM96 & EIGEN-GRACE02S & EIGEN-CG01C & GGM02S \\
\hline 2 & $3.561 \times 10^{-11}$ & $5.304 \times 10^{-11}$ & $3.750 \times 10^{-11}$ & $1.1 \times 10^{-10}$ \\
4 & $1.042 \times 10^{-10}$ & $3.921 \times 10^{-12}$ & $6.242 \times 10^{-12}$ & $8.3 \times 10^{-12}$ \\
6 & $1.449 \times 10^{-10}$ & $2.049 \times 10^{-12}$ & $2.820 \times 10^{-12}$ & $4.5 \times 10^{-12}$ \\
8 & $2.266 \times 10^{-10}$ & $1.479 \times 10^{-12}$ & $1.792 \times 10^{-12}$ & $2.8 \times 10^{-12}$ \\
10 & $3.089 \times 10^{-10}$ & $2.101 \times 10^{-12}$ & $1.317 \times 10^{-12}$ & $2 \times 10^{-12}$ \\
12 & $4.358 \times 10^{-10}$ & $1.228 \times 10^{-12}$ & $1.053 \times 10^{-12}$ & $1.8 \times 10^{-12}$ \\
14 & $5.459 \times 10^{-10}$ & $1.202 \times 10^{-12}$ & $8.931 \times 10^{-13}$ & $1.6 \times 10^{-12}$ \\
16 & $5.313 \times 10^{-10}$ & $9.945 \times 10^{-13}$ & $7.905 \times 10^{-13}$ & $1.6 \times 10^{-12}$ \\
18 & $4.678 \times 10^{-10}$ & $9.984 \times 10^{-13}$ & $7.236 \times 10^{-13}$ & $1.6 \times 10^{-12}$ \\
20 & $4.690 \times 10^{-10}$ & $1.081 \times 10^{-12}$ & $6.784 \times 10^{-13}$ & $1.6 \times 10^{-12}$ \\
\hline
\end{tabular}

one new satellite, its data should be combined with those of the existing LAGEOS and LAGEOS II. While the gravitational error could be pushed below the $1 \%$ level by the forthcoming, more robust solutions for the terrestrial gravitational field, the error due to the non-gravitational perturbations would remain $\sim 1 \%$ due to their impact on the LAGEOS satellites. However, the implementation of such a mission would be much facilitated by the fact that an orbit lower than that of LAGEOS, as in the original LAGEOSLARES experiment, would be acceptable for the new laser target.

Only the launch of at least two new spacecraft endowed with some active mechanism of compensation of the non-gravitational perturbations could allow to discard the passive LAGEOS satellites and, then, to reduce the total systematic error below the $1 \%$ threshold fully exploiting the benefits from the CHAMP and GRACE models. Of course, this option would be, in practice, much less feasible because of its higher cost. 


\section{What can be done with only one new, passive satellite of LAGEOS type?}

In this Section we will analyze the impact of the new Earth gravity model solutions from the dedicated CHAMP and GRACE missions on a scenario in which only one new passive satellite of LAGEOS type is at our disposal. We will show that it is not possible to only use its data. Instead, a suitable combination of them with those of the existing LAGEOS satellites would allow to obtain a robust and reliable measurement of the Lense-Thirring effect at the $\sim 1 \%$ level with a lower-cost mission with respect to the originally proposed LARES orbital configuration. Indeed, in regard to the semimajor axis, crucial for the cost of the mission whose most expensive part is represented by the launcher, it would be possible to pass from $12270 \mathrm{~km}$ to $7-8000 \mathrm{~km}$ only. The Earth gravity model EIGEN-CG01C has been adopted.

\subsection{The originally proposed LAGEOS-LARES scenario: the sum of the nodes}

The originally proposed LAGEOS-LARES/LAGEOS III mission (Ciufolini 1986) can only be realized if the LARES satellite has its orbital parameters as closest as possible to those of the existing LAGEOS, with the inclination $180 \mathrm{deg}$ apart. Such strict constraints would make the launch of LARES more demanding in terms of quality, power and cost of the launcher. This could become a serious drawback for a specifically dedicated space mission which could improve the present-day accuracy in knowing the Lense-Thirring effect of more or less one order of magnitude with respect to the already performed or proposed tests with the existing satellites. Indeed, the use of the data from the old-generation passive LAGEOS satellite would keep the systematic error due to the non-gravitational perturbations at the 1$2 \%$ level, irrespectively of what the improvement in the systematic error of gravitational origin could be.

Another important point is that the simple sum of the nodes of two satellites, by construction, does not cancel out any even zonal harmonic: an exact cancellation occurs only if the semimajor axis and the eccentricities are identical and the inclinations are $180 \mathrm{deg}$ apart. Since this cannot be realized in the real world, the sum of the nodes remains exposed to the residual

corrupting action of all the even zonal harmonics of the geopotential and of their secular variations. This topic has been addressed in (Iorio 2003a) with the EGM96 model. 
In Figure 1 we show the impact of the unavoidable orbital injection errors in the semimajor axis according to the EIGEN-CG01C model. It can be noted that, despite the improvements in our knowledge of the terrestrial gravity field, the orbital height of LARES should still be similar to that of LAGEOS.

In Figure 2 the impact of the departures of the inclination from its nominal value is shown. Again, the need for a high accuracy in inserting LARES in orbit with an inclination as closest as possible to its nominal value is apparent.

It must be pointed out that in Figure 11 and Figure 2 also the impact of the secular variations of the low-degree even zonal harmonics $\dot{J}_{2}, \dot{J}_{4}, \dot{J}_{6}$ has been included and calculated over 10 years. Since their integrated shift grows quadratically in time, their corrupting impact would become relatively important over observational time spans some years long due to the orbital injection errors.

\subsubsection{Conclusions}

In conclusion, the originally proposed LAGEOS-LARES scenario could work only with a relatively expensive mission mainly due to the need of a launcher of high performance and quality in order to reduce the orbital injection errors. The total accuracy would amount to a few percent and would depend on the precision with which the nominal orbit could be realized in practice. Moreover, the secular variations of all the low-degree even zonal harmonics would further corrupt the recovery of the Lense-Thirring precessions over observational time spans some years long. In view of the already performed measurements and of the suggested alternative tests with the existing satellites the originally proposed LAGEOS-LARES two-nodes scenario appears now difficult to be supported.

\subsection{The LARES-only scenario}

In this Section we will examine the possibility of only using the node of LARES in order to exploit its reduced sensitivity to the non-gravitational perturbations thanks to its proposed particular construction. More precisely, we want to see if discarding the LAGEOS data could allow to push the total systematic error below the 1\% level. The EIGEN-CG01C Earth gravity model is adopted in order to assess the impact of the classical part of the terrestrial gravitational field. Unfortunately, the answer is negative. 

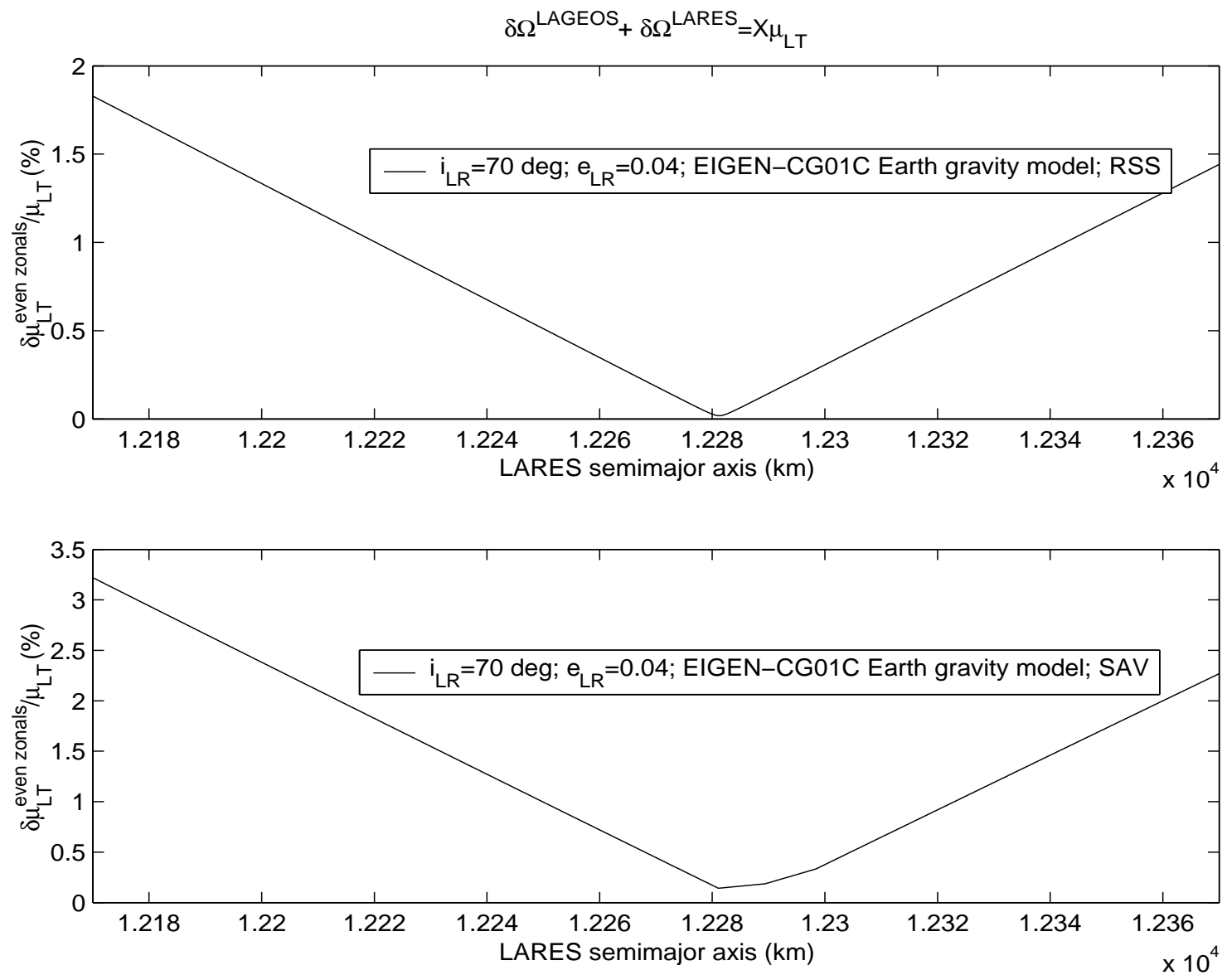

Figure 1: Dependence on $a$ of the percent 1-sigma systematic error $\delta \mu_{\mathrm{LT}}^{\text {even zonals }} / \mu_{\mathrm{LT}}$ due to the mismodelling in the static part of the even zonal harmonics of the geopotential according to EIGEN-CG01C for the sum of the residuals of the nodes $\delta \Omega^{\mathrm{LAGEOS}}+\delta \Omega^{\mathrm{LARES}}$ of the originally proposed LAGEOS-LARES configuration with $i=70 \mathrm{deg} \mathrm{km}$ and $e=0.04$. The secular variations of the even zonal harmonics $\dot{J}_{2}, \dot{J}_{4}, \dot{J}_{6}$ have been included. The chosen time span is 10 years. In the upper panel the Root-Sum-Square (RSS) calculation is presented, while in the lower panel the linear Sum of the Absolute Values (SAV) of the individual errors is shown. 

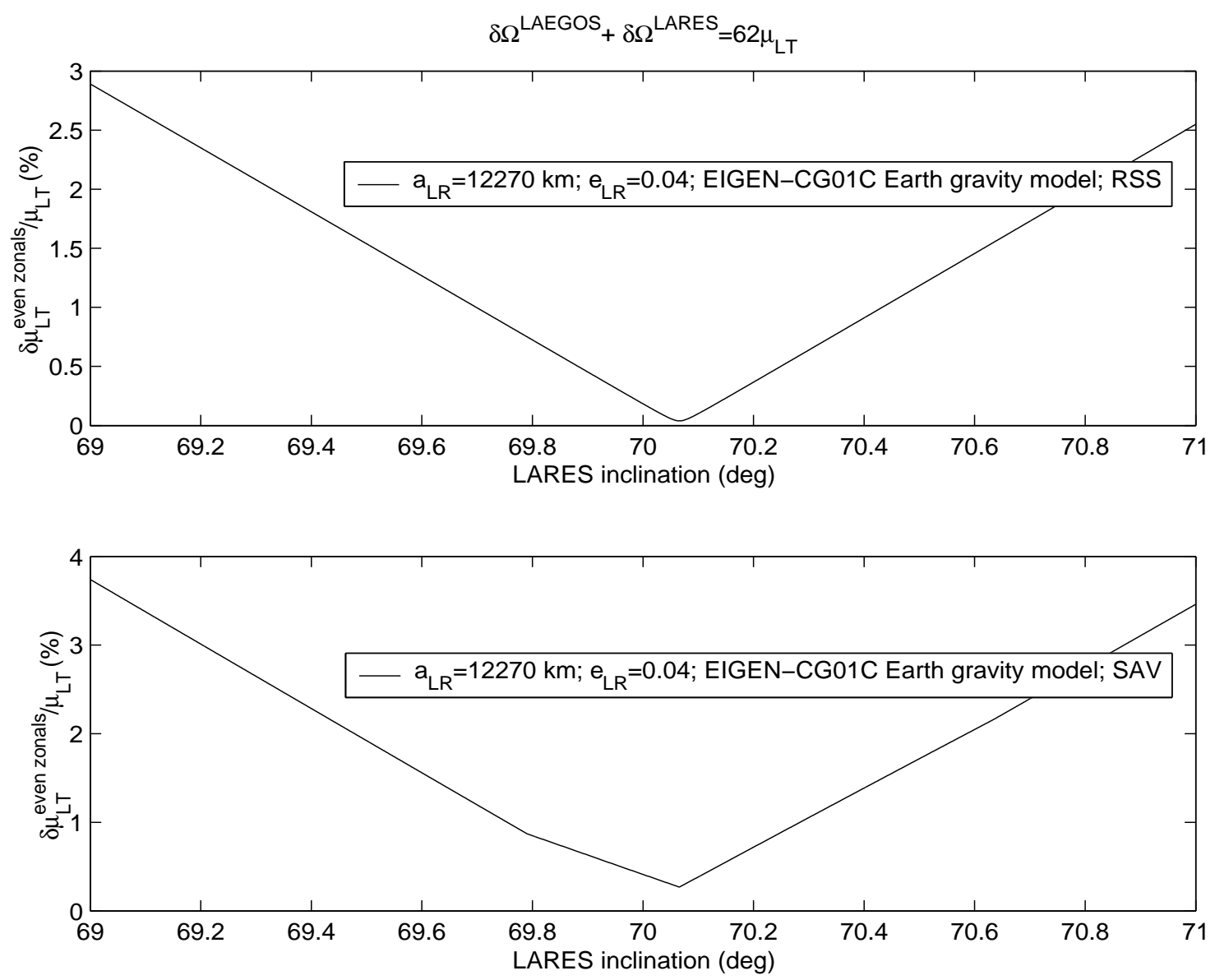

Figure 2: Dependence on $i$ of the percent 1-sigma systematic error $\delta \mu_{\mathrm{LT}}^{\text {even zonals }} / \mu_{\mathrm{LT}}$ due to the mismodelling in the static part of the even zonal harmonics of the geopotential according to EIGEN-CG01C for sum of the residuals of the nodes $\delta \Omega^{\mathrm{LAGEOS}}+\delta \Omega^{\mathrm{LARES}}$ of the originally proposed LAGEOS-LARES configuration with $a=12270 \mathrm{~km}$ and $e=0.04$. The secular variations of the even zonal harmonics $\dot{J}_{2}, \dot{J}_{4}, \dot{J}_{6}$ have been included. The chosen time span is 10 years. In the upper panel the Root-Sum-Square (RSS) calculation is presented, while in the lower panel the linear Sum of the Absolute Values (SAV) of the individual errors is shown. 


\subsubsection{The nearly-polar orbit}

The 1-sigma error due to the geopotential, according to EIGEN-CG01C, for a nearly-polar orbital geometry is depicted in Figure 3. For $i=89.9$ deg the systematic error due to the geopotential amounts to $1-2 \%$; unfortunately, the period of the node, which is also the period of the gravitational perturbation due to the solar $K_{1}$ tide, amounts to $\sim 10^{4}$ days. As already shown in (Peterson 1997, Iorio 2002a, 2005b), the mismodelled part of such a tidal perturbation would act as a huge superimposed bias on the Lense-Thirring trend over a reasonable observational time span of some years. On the other hand, for $i=88 \mathrm{deg}$ the node period would reduce to $\sim 10^{3}$ days but the systematic error due to the mismodelling in the static part of geopotential would amount to $15-25 \%$.

A further source of uncertainty is also represented by the impact of the secular variations $\dot{J}_{\ell}$ of the low-degree even zonal harmonics whose shift on the node grows quadratically in time.

\subsubsection{The non-polar scenario}

In order to avoid the problems with the $K_{1}$ tide an inclination far from $90 \mathrm{deg}$ could, in principle, be adopted. Indeed, for $i=70 \mathrm{deg}$ the period of the node would amount to $\sim 10^{2}$ days. However, for non polar-orbital geometries the mismodelling in the static part of the geopotential would be fatal independently of the chosen semimajor axis, as depicted in Figure 4. The secular variations $\dot{J}_{\ell}$ of the low degree even zonal harmonics would further corrupt the measurement of the Lense-Thirring effect.

\subsubsection{Conclusions}

These considerations clearly show that the possibility of only analyzing the node of a suitably built passive satellite is not viable because of the impact of all the uncancelled even zonal harmonics of the geopotential along with their secular variations. The polar geometry would allow to reduce the consequences of these sources of error but, on the other hand, it would enhance the aliasing effect of the tidal perturbations due to the $K_{1}$ tide whose period is equal to that of the node which would amount to $10^{3}-10^{4}$ days. 

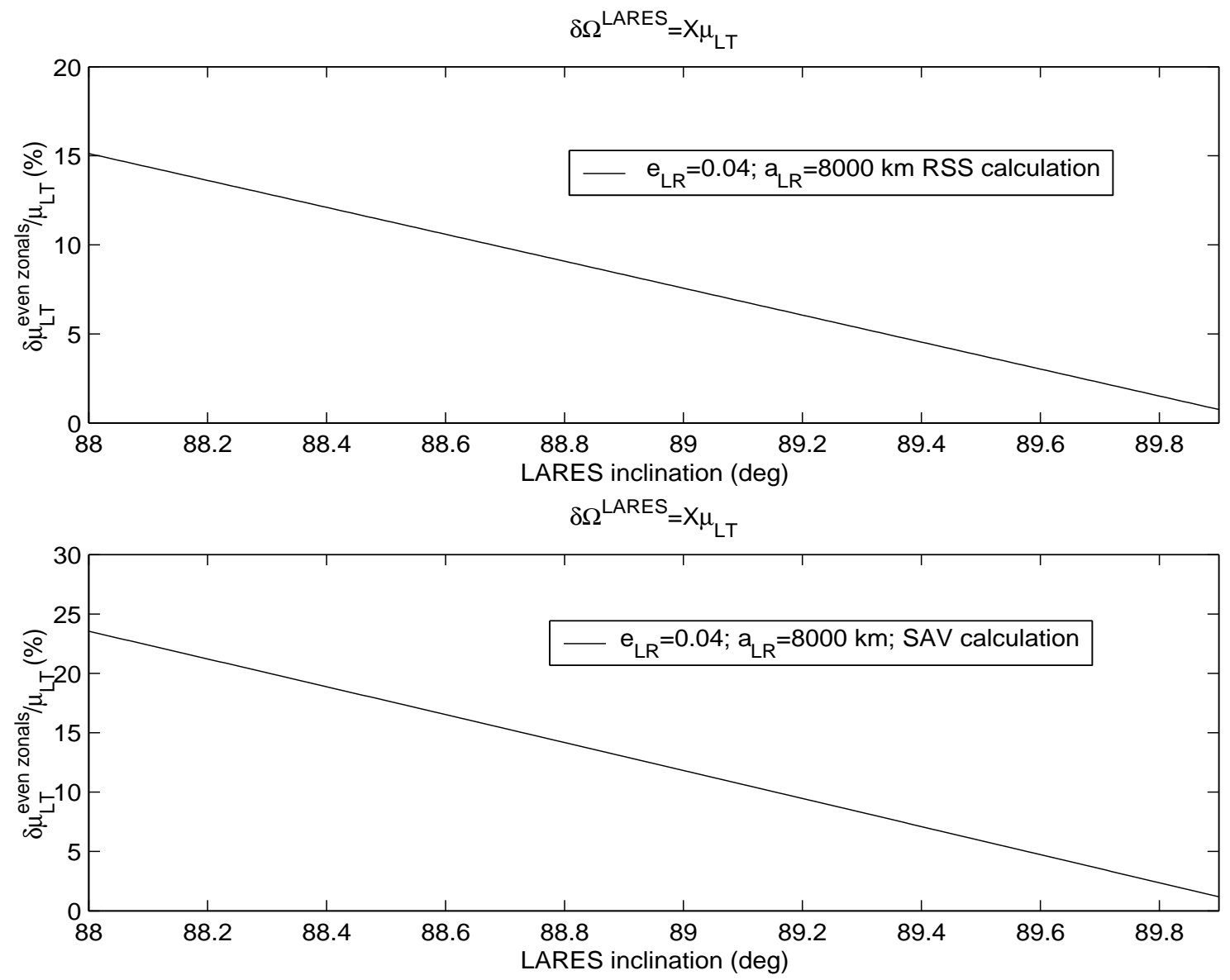

Figure 3: Dependence on $i$ of the percent 1-sigma systematic error $\delta \mu_{\mathrm{LT}}^{\text {even zonals }} / \mu_{\mathrm{LT}}$ due to the mismodelling in the static part of the even zonal harmonics of the geopotential according to EIGEN-CG01C for the residuals of the node $\delta \Omega^{\mathrm{LARES}}$ of a nearly-polar orbital configuration with $a=8000 \mathrm{~km}$ and $e=0.04$. The secular variations of the even zonal harmonics $\dot{J}_{2}, \dot{J}_{4}, \dot{J}_{6}$ has not been included. In the upper panel the Root-Sum-Square (RSS) calculation is presented, while in the lower panel the linear Sum of the Absolute Values (SAV) of the individual errors is shown. 

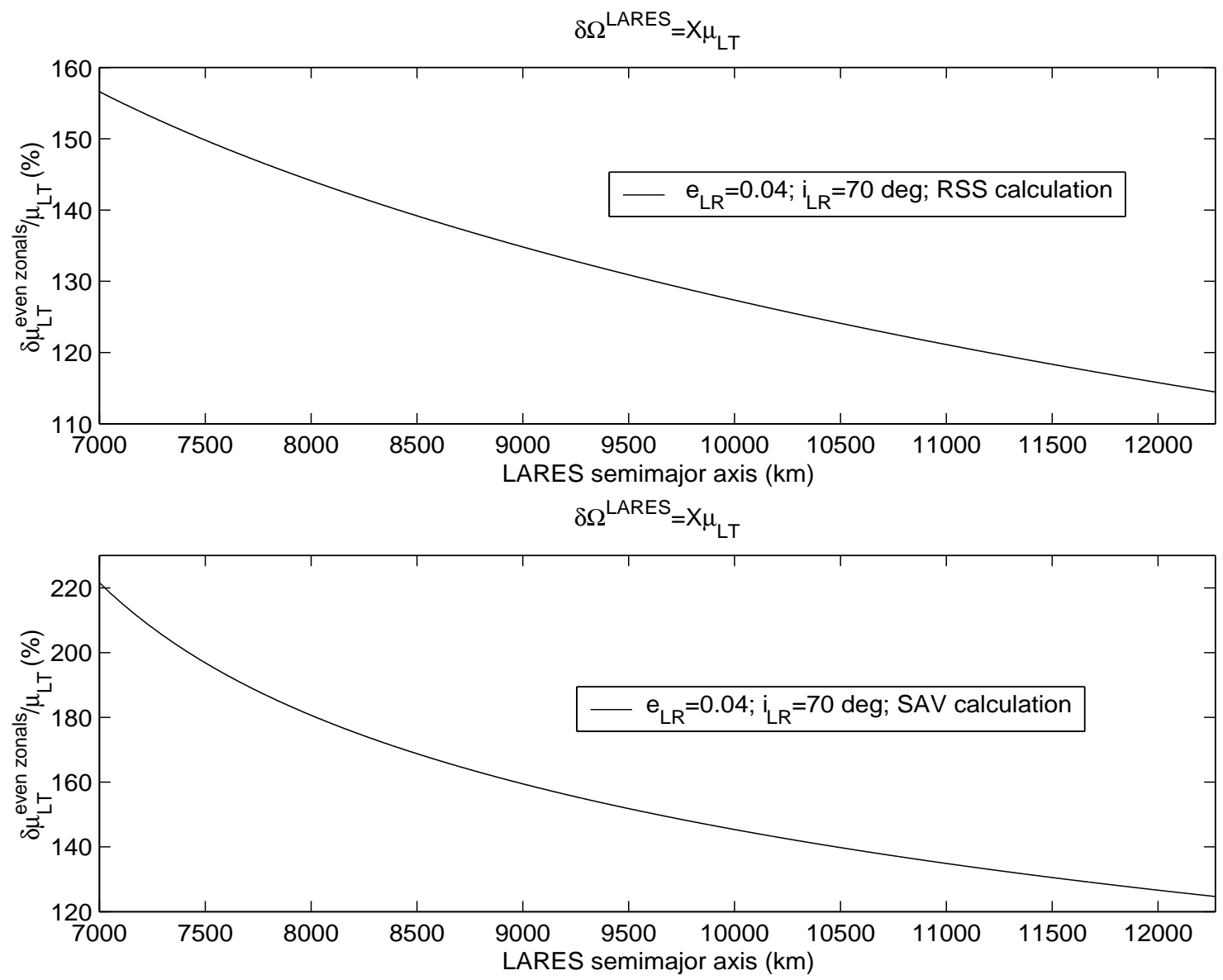

Figure 4: Dependence on $a$ of the percent 1-sigma systematic error $\delta \mu_{\mathrm{LT}}^{\text {even zonals }} / \mu_{\mathrm{LT}}$ due to the mismodelling in the static part of the even zonal harmonics of the geopotential according to EIGEN-CG01C for the residuals of the node $\delta \Omega^{\text {LARES }}$ of a non-polar orbital configuration with $i=70 \mathrm{deg}$ and $e=0.04$. The secular variations of the even zonal harmonics $\dot{J}_{2}, \dot{J}_{4}, \dot{J}_{6}$ has not been included. In the upper panel the Root-Sum-Square (RSS) calculation is presented, while in the lower panel the linear Sum of the Absolute Values (SAV) of the individual errors is shown. 


\subsection{A high-altitude LARES scenario}

For the sake of completeness, we examine the possibility of adopting for the new satellite a semimajor axis much larger than that of LAGEOS. E.g., a value $a=36000 \mathrm{~km}$ has been suggested in (Ciufolini 2004). Apart from the fact that such a mission would become even more expensive than the originally proposed LAGEOS-LARES scenario, it would be unfeasible because of the geopotential's mismodelling.

Indeed, the node of such a high-altitude satellite could not be combined with those of LAGEOS and LAGEOS II because it would be weighted by a quite large coefficient which would enhance the time-dependent perturbations of gravitational and non-gravitational origin. Among the tidal perturbations, that induced by the $K_{1}$ tide would be fatal because its period, equal to that of the satellite's node, would be $\sim 10^{4}$ days long. Such issues have been addressed in (Iorio 2002b, Vespe and Rutigliano 2004).

Also the possibility of using only the node is not viable. Indeed, for such

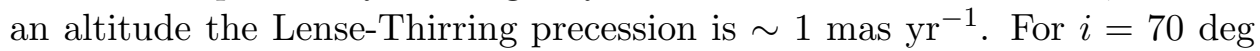
and $e=0.04$ the sum of the mismodelled precessions induced by the even zonal harmonics would be $\sim 100 \%$ of the gravitomagnetic effect mainly due to $J_{2}$ and $J_{4}$, according to EIGEN-CG01C. For $i=89 \mathrm{deg}$ and $e=0.04$ the impact of the static part of the geopotential would be reduced to $5 \%$ of the Lense-Thirring effect, but the $K_{1}$ tide would induce an aliasing semisecular perturbation with a period of $10^{5}$ days.

\subsection{The linear combination scenario with LAGEOS and LA- GEOS II}

The most viable approach with only one new dedicated passive satellite at our disposal would be the suitable combination of its data with those of the already existing LAGEOS and LAGEOS II. Such proposal was already put forth in (Iorio et al. 2004) in the framework of the relativity-dedicated OPTIS mission (Lämmerzahl et al. 2004) by retaining for the new satellite the nominal configuration of LARES. A previous analysis involving also the perigee of LARES and EGM96 can be found in (Iorio et al. 2002). According to a well established procedure (Ciufolini 1996; Iorio and Morea 2004, Iorio 2004, Vespe and Rutigliano 2004), it is possible to design a linear combination of the residuals of the nodal rates of LAGEOS, LAGEOS II and LARES

$$
\delta \dot{\Omega}^{\mathrm{LAGEOS}}+c_{1} \delta \dot{\Omega}^{\mathrm{LAGEOSII}}+c_{2} \delta \dot{\Omega}^{\mathrm{LARES}} \sim X_{\mathrm{LT}} \mu_{\mathrm{LT}}
$$


which would cancel out the first two even zonal harmonics of the geopotential along with their temporal variations. The parameter $\mu_{\mathrm{LT}}$ is 0 in the Newtonian mechanics and 1 in the General Theory of Relativity. The coefficients can be found in Figure 5 and the slope of the gravitomagnetic trend in Figure 6] If, on the one hand, the inclusion of the existing LAGEOS satellites would prevent to go below the $1 \%$ level due to the non-gravitational systematic errors, on the other hand such goal could be reached with a much less expensive mission because, as we will show, the constraints on the new satellite's orbit could be greatly weakened thanks to the new Earth gravity models from CHAMP and GRACE. It would be possible to choose a much smaller semimajor axis and also the inclination could safely range a wider span near $70 \mathrm{deg}$ without sensibly affecting the systematic error due to the uncancelled even zonal harmonics of the geopotential. Another important advantage is that the secular variations $\dot{J}_{2}, \dot{J}_{4}$ would not affect this combination.

In Figure 7 the dependence of the systematic error due to the geopotential on the semimajor axis is presented. It is important to note that even with a semimajor axis of $7500-8000 \mathrm{~km}$ the present-day mismodelling in the even zonal harmonics of the geopotential the $1 \%$ level in their impact could be easily reached. Note also that for such an altitude a calculation of the systematic error of gravitational origin up to degree $\ell=20$ is well adequate and reliable: indeed, it can be shown that the result does not change by adding further terms of higher degree. The forthcoming more robust and reliable Earth gravity models from CHAMP and GRACE should further improve this situation. Moreover, no semisecular time-dependent perturbations would be introduced: indeed, the period of the node of LARES would amount to $\sim 10^{2}$ days for $a=7500-8000 \mathrm{~km}$.

In regard to the departure of the inclination from the nominal value of 70 deg, Figure 8, obtained for $a=8000 \mathrm{~km}$, clearly shows that the systematic bias of gravitational origin is rather insensitive to the orbital injection errors over a wide range. However, it must be noted that an analysis conducted from $0 \mathrm{deg}$ to $180 \mathrm{deg}$ has revealed that the coefficients $c_{1}$ of LAGEOS II and $c_{2}$ of the new satellite become $\gg 1$ for $i \sim 50$ and $129 \mathrm{deg}$, while for a polar orbit $c_{2}$ blows up.

The EUROCKOT facilities at Plesetsk cosmodrome, located at latitude $62.7 \mathrm{deg} \mathrm{N}$, would easily allow to get the inclination $i=75.3 \mathrm{deg}$ for both circular and elliptical orbits in the altitude range from $200 \mathrm{~km}$ up to 2000 km (see Chapter 3 General Performance Capabilities of the EUROCKOT User Guide http://www.eurockot.com/pb-pic/20041084.pdf).

Note that Figure $[5$ yields another possible reason to further reduce the 

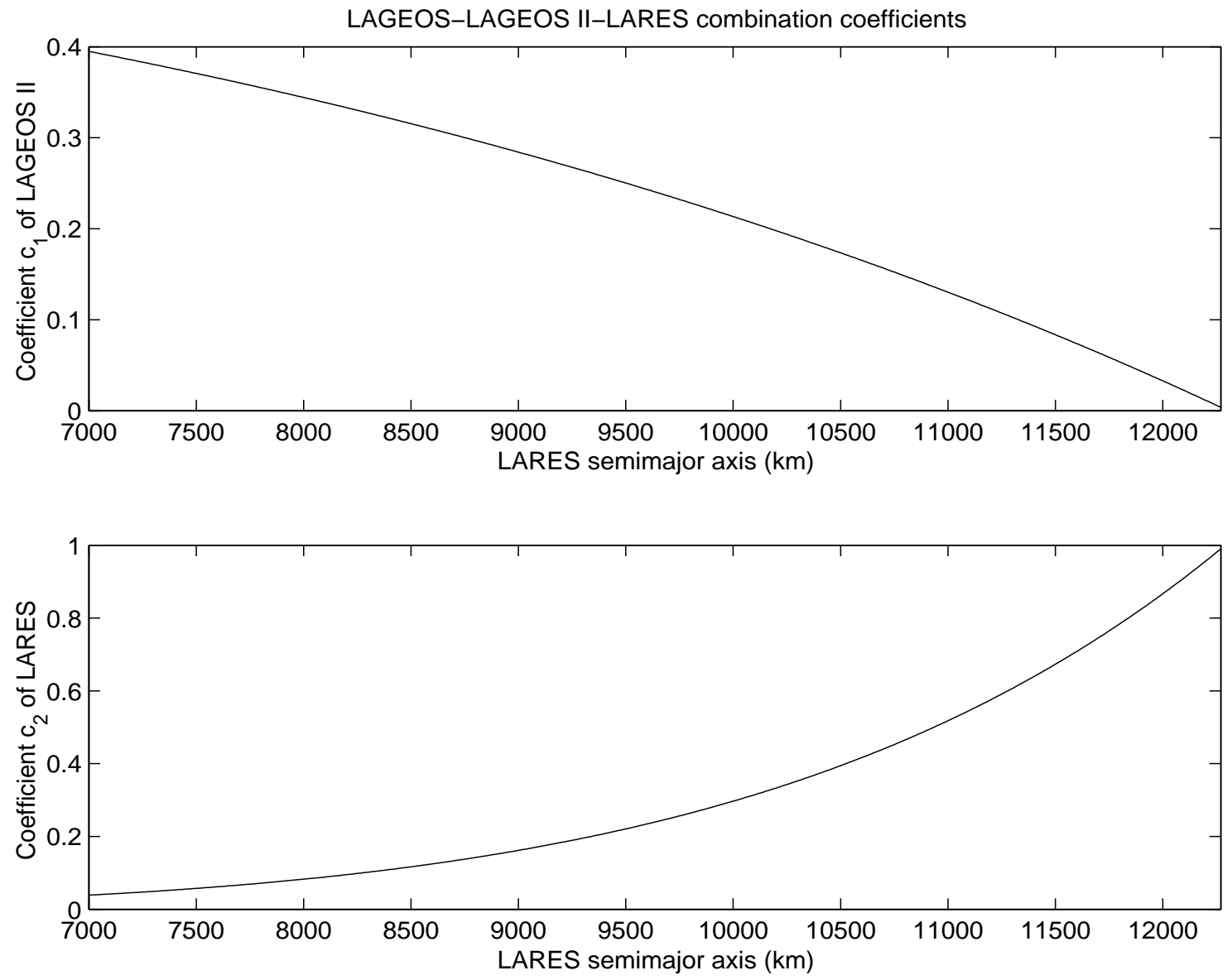

Figure 5: Dependence on $a$ of the coefficients $c_{1}$ and $c_{2}$ with which the nodes of LAGEOS II and LARES enter the combination of eq. (2). The values $i=70 \mathrm{deg}$ and $e=0.04$ have been assumed. 


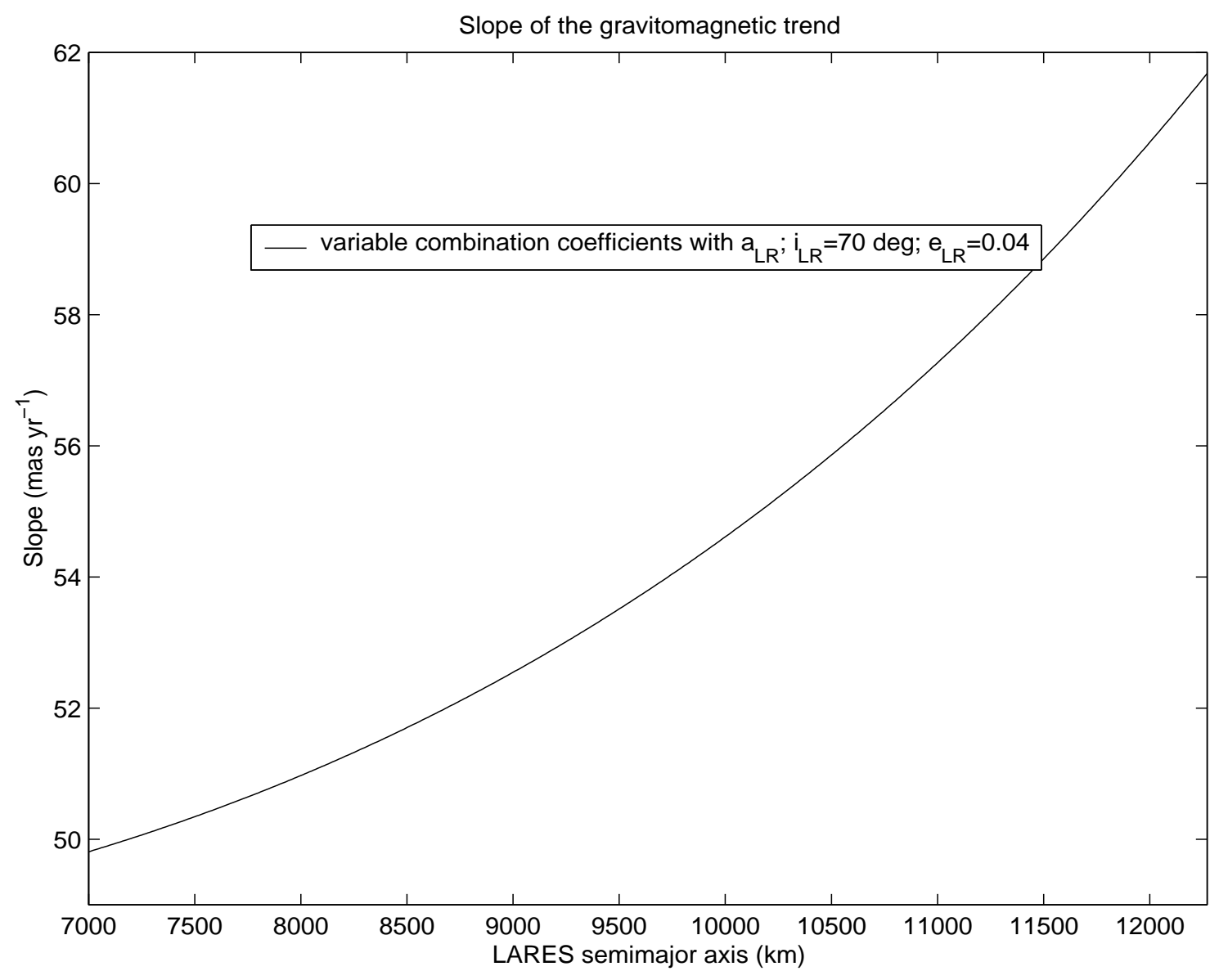

Figure 6: Dependence on $a$ of the slope $X_{\mathrm{LT}}$, in mas $\mathrm{yr}^{-1}$, of the gravitomagnetic trend of the combination eq. (2). 


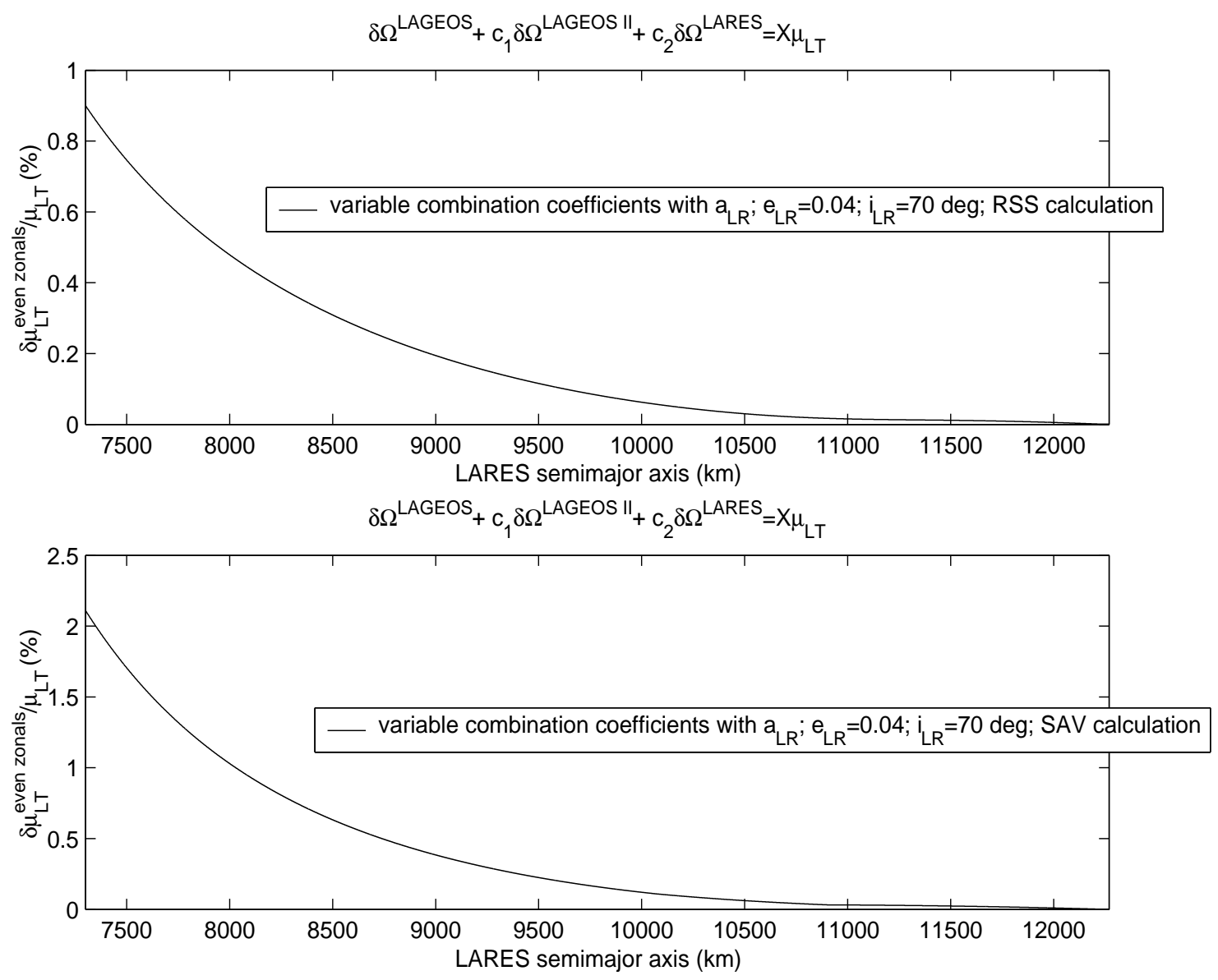

Figure 7: Dependence on $a$ of the percent 1-sigma systematic error $\delta \mu_{\mathrm{LT}}^{\text {even zonals }} / \mu_{\mathrm{LT}}$ due to the mismodelling in the static part of the even zonal harmonics of the geopotential according to EIGEN-CG01C for a linear combination $\delta \Omega^{\mathrm{LAGEOS}}+c_{1} \delta \Omega^{\mathrm{LAGEOS} \text { II }}+c_{2} \delta \Omega^{\mathrm{LARES}}$ with the existing LAGEOS and LAGEOS II with $i_{\mathrm{LR}}=70 \mathrm{deg}$. In the upper panel the Root-SumSquare (RSS) calculation is presented, while in the lower panel the linear Sum of the Absolute Values (SAV) of the individual errors is shown. 

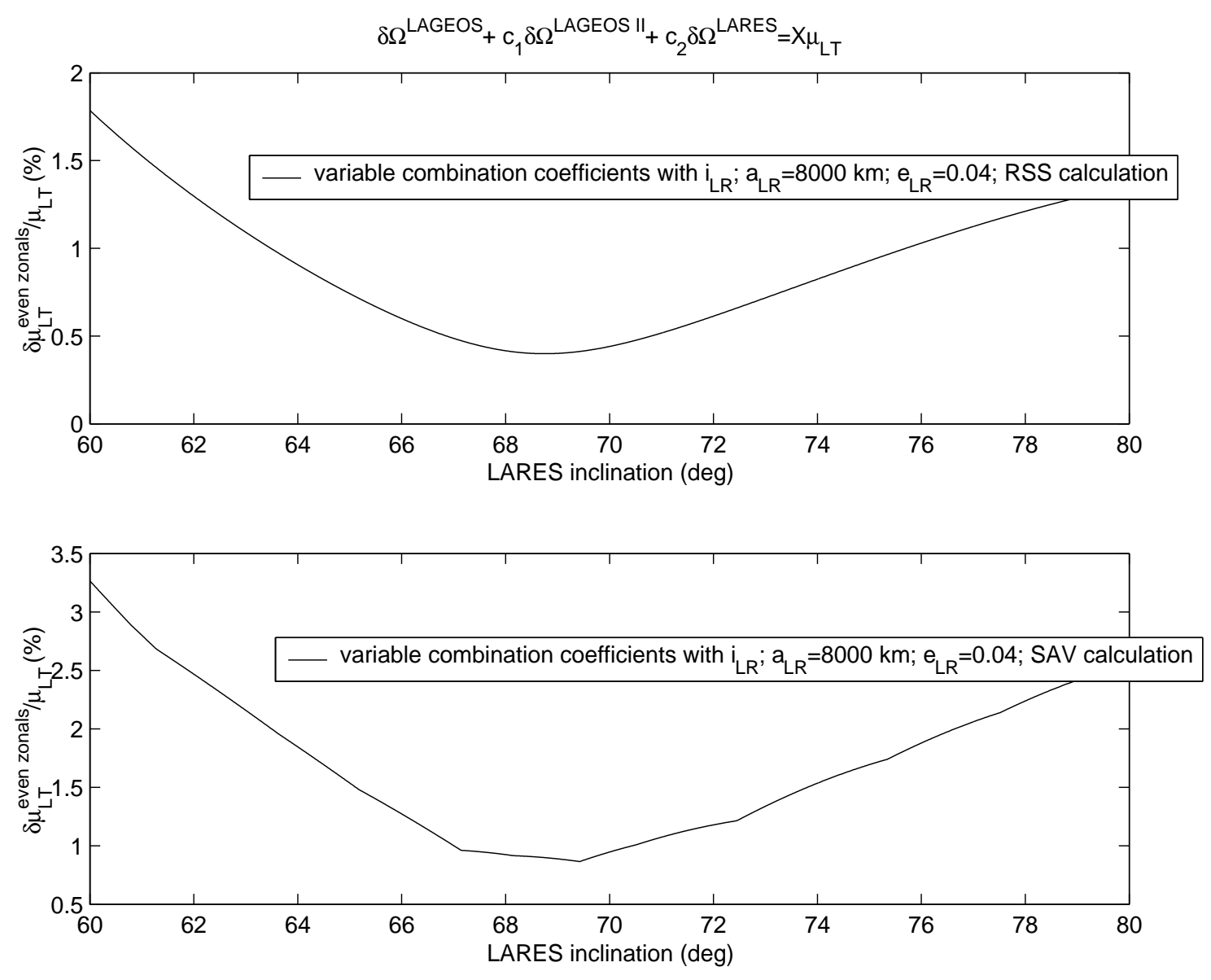

Figure 8: Dependence on $i$ of the percent 1-sigma systematic error $\delta \mu_{\mathrm{LT}}^{\text {even zonals }} / \mu_{\mathrm{LT}}$ due to the mismodelling in the static part of the even zonal harmonics of the geopotential according to EIGEN-CG01C for a linear combination $\delta \Omega^{\mathrm{LAGEOS}}+c_{1} \delta \Omega^{\mathrm{LAGEOS} I \mathrm{I}}+c_{2} \delta \Omega^{\mathrm{LARES}}$ with the existing LAGEOS and LAGEOS II with $a_{\mathrm{LR}}=8000 \mathrm{~km}$. In the upper panel the Root-SumSquare (RSS) calculation is presented, while in the lower panel the linear Sum of the Absolute Values (SAV) of the individual errors is shown. 
cost of the mission: indeed, the coefficient $c_{2}$ with which the node of LARES enters the combination of eq. (2) becomes smaller than 0.1 for low altitudes. This means that, if one is only interested in the node for the Lense-Thirring effect, it would not be necessary a particular technological and financial effort in building the satellite in order to reduce the action of the nongravitational perturbations with respect to the level of the existing LAGEOS satellites.

Finally, one may ask if the combination of eq. (2) could be implemented with some of the other existing laser-tracked satellites: after all, the majority of them orbit at altitudes much lower than LAGEOS and LAGEOS II and for some of them the centimeter accuracy in reconstructing their orbits has been obtained. The most suitable candidate seems to be Ajisai, whose orbital parameters are $a=7870 \mathrm{~km}, e=0.001$ and $i=50 \mathrm{deg}$. Unfortunately, it turns out that its inclination lies in a domain in which the systematic error due to the even zonal harmonics would be unacceptably large: indeed, it would amount to $\sim 20 \%$ according to EIGEN-CG01C.

\section{What can be done with two new spacecraft?}

The possibility of using more than one new passive laser-ranged satellite together with the existing LAGEOS and LAGEOS II was investigated for the first time in (Peterson 1997).

In (Iorio 2003b, 2003c, Iorio and Lucchesi 2003) it has been shown that for a pair of satellites in supplementary orbital configuration also the differences of the perigees could be used in addition to the sum of the nodes. Indeed, the Lense-Thirring perigee precessions depend on $\cos i$, while the aliasing classical precessions induced by the even zonal harmonics of the geopotential are of the form (Iorio 2003d)

$$
\dot{\omega}^{\left(J_{\ell}\right)}=A^{(\ell)} \cos ^{2} i\left[\sum_{k=2}^{\ell} p_{k}^{(\ell)} \sin ^{k} i+B^{(\ell)}\right]+C^{(\ell)}\left[\sum_{h=2}^{\ell} q_{h}^{(\ell)} \sin ^{h} i\right]+D^{(\ell)}
$$

with $h, k$ even, $p_{k}^{(\ell)}, q_{k}^{(\ell)}$ numerical coefficients which depend only on the degree $\ell$ and $A^{(\ell)}, B^{(\ell)}, C^{(\ell)}, D^{(\ell)}$ different functions of $a$ and $e$ for the various degrees $\ell$. Then, with the differences of the perigees for identical orbital configurations and supplementary inclinations the gravitomagnetic precessions add up and all the classical precessions cancel out. Such observable could not be implemented by using many of the already existing SLR satellites due to the small eccentricities of their orbits: in (Iorio 2003b) it has 
been shown that LAGEOS II would not be suitable for implementing the difference of the perigee because of certain long-period perturbations of gravitational and non-gravitational origin which affect its perigee ${ }^{1}$. In (Iorio and Lucchesi 2003) a configuration of two new passive satellites of LAGEOS-type in eccentric orbits has been investigated: it turned out that the difference of the perigees would be affected at a $\sim 5 \%$ level, mainly due to the impact of the non-gravitational perturbations. A $\sim 1 \%$ level could be achievable with the sum of the nodes, but it is clear that the implementation of the supplementary orbital configuration with a pair of passive spacecraft would not be competitive with that previously analyzed which would allow to reach the same goal-a $\sim 1 \%$ measurement of the Lense-Thirring effect-with just one new satellite.

Instead, if an active drag-free technology was used, the implementation of the supplementary configuration with two satellites would be very interesting. Indeed, both the nodes and the perigees would be available and it would be possible to analyze the sum of the nodes, the difference of the perigees and some suitable linear combination of them without resorting to the existing LAGEOS and LAGEOS II. In this way the Lense-Thirring effect could be measured by reducing the total systematic error below the $1 \%$ level and fully exploiting the benefits of the CHAMP and GRACE gravity models.

Of course, the cost of such an option would be much higher than those required to built just one passive laser target and insert it into a relatively low orbit.

\section{Conclusions}

The notable improvements in our knowledge of the classical part of the terrestrial gravitational potential due to the dedicated CHAMP and, especially, GRACE missions would make the launch of a third satellite of LAGEOStype dedicated to the measurement of the Lense-Thirring effect cheaper than it has been considered until now. Indeed, while the originally proposed scenario involving the analysis of the sum of the nodes of LAGEOS and LARES would be relatively expensive to be implemented due to the need of obtaining the same high altitude of LAGEOS, it would, instead, be possible to suitably combine the data of the new satellite with those of the

\footnotetext{
${ }^{1}$ In (Peterson 1997) it has also been shown that neither the sum of the nodes would be a viable opportunity if LAGEOS II was one of the partners of the supplementary configuration.
} 
existing LAGEOS and LAGEOS II in order to cancel out $J_{2}$ and $J_{4}$ along with their temporal variations abandoning the originally proposed stringent requirements on the orbital geometry of the new laser target. In particular, it would be possible to reduce the semimajor axis-and, then, the cost of the launcher-from that of LAGEOS, i.e. $12270 \mathrm{~km}$, down to $7500-8000 \mathrm{~km}$ keeping the systematic error due to the mismodelled even zonal harmonics of the geopotential within the $1 \%$ level (at 1-sigma) according to the EIGEN-CG01C Earth gravity model. Although the optimal inclination still remains close to $70 \mathrm{deg}$, departures of many degrees from such a value are now well acceptable. For a combination with LAGEOS and LAGEOS II the only forbidden windows are near 50, 90 and 130 deg. Moreover, no semisecular long-period perturbations would be introduced because the period of the node of the new satellite would be of the order of $10^{2}$ days. Finally, with the low-altitude option it would also be possible to save money on the construction of the satellite because it would not be necessary to greatly reduce the impact of the non-gravitational perturbations with respect to the level of the existing LAGEOS satellites thanks to the small value $(\leq 0.1)$ of the coefficient with which the new object would enter the combination. In this way the realization of a cheap space mission dedicated to the measurement of the Lense-Thirring effect with a $\sim 1 \%$ level of accuracy becomes much easier, at least in principle. Such a precision is better than what could be obtained by reanalyzing the data of the existing satellites. Moreover, the problems with the secular variations of the even zonal harmonics which affect the performed test with the LAGEOS-LAGEOS II two-node combination would greatly be reduced. On the other hand, one could legitimately ask if such an expense is justified for a satellite whose principal (or unique) goal is the measurement of the Lense-Thirring effect, while with the already performed or proposed tests with the existing satellites the obtainable accuracy in testing the gravitomagnetic effect on the orbit of a test particle is only ten-twenty times worse. If, instead, it was possible to also include this goal in the list of the scientific objectives of other already planned or proposed space missions, like e.g. OPTIS, without notably increasing their costs or even suggesting ways to reduce them, this would be a major achievement. E.g. it seems that a new geodetic satellite at a low-medium altitude might be useful for many geodetic and geodynamic purposes like a better knowledge of the centre-of-mass of our planet (Ries 2005, private communication). In regard to OPTIS, the minimum orbital height should be $\sim 1000 \mathrm{~km}$, i.e. $a \sim 7378 \mathrm{~km}$, due to the limitations posed by the drag-free technology to be adopted based on the field emission electrical propulsion (FEEP): this requirement would be compatible with the lower altitude scenario outlined 
in the paper.

Since it would not be possible to use the data only from such a new satellite, the fact that they should be combined with those from LAGEOS and LAGEOS II sets the limit of the obtainable systematic error to $\sim 1 \%$ due to the action of the non-gravitational perturbations on the existing twins, irrespectively of the fact that the gravitational error could be reduced well below such a cutoff. Only the launch of at least two new satellites endowed with some active mechanism of compensation of the non-conservative accelerations and in rather elliptic orbits would allow to discard the existing LAGEOS satellites fully exploiting the possibilities opened by the new Earth gravity models from CHAMP and GRACE and pushing the total systematic error below the $1 \%$ level.

In conclusion, a reliable $1 \%$ measurement of the Lense-Thirring effect could be obtained with a new satellite placed in a relatively low orbit ( $a=$ $7500-8000 \mathrm{~km}$ ) which would allow to greatly reduce the costs with respect to, e.g., the originally proposed LARES mission. The node of such a new satellite, which could also be passive and built without resorting to any particularly sophisticated technologies, should be combined with those of LAGEOS and LAGEOS II in order to cancel out the first two even zonal harmonics along with their secular variations. The limit of the reduction of the systematic error is set by the non-gravitational perturbations acting on the existing twin satellites, irrespectively of the improvements allowed by the new forthcoming CHAMP/GRACE-based Earth gravity models. A total systematic error smaller than $1 \%$ could be obtained with at least two new drag-free spacecraft.

\section{Acknowledgments}

I am grateful to J. Ries for useful discussions.

\section{References}

Ciufolini, I., Measurement of Lense-Thirring drag on high-altitude laser ranged artificial satellite, Phys. Rev. Lett., 56, 278-281, 1986.

Ciufolini, I., On a new method to measure the gravitomagnetic field using two orbiting satellites, Il Nuovo Cimento A, 109, 1709-1720, 1996. 
Ciufolini, I., The Concept of the LARES experiment, in: LARES Phase-A Study, pp. 16-33, Rome, 1998.

Ciufolini, I., LARES/WEBER-SAT, frame-dragging and fundamental phyiscs, preprint http://www.arxiv.org/abs/gr-qc/0412001, 2004.

Ciufolini, I., Pavlis, E. C., Chieppa, F., Fernandes-Vieira, E., and Pérez-Mercader, J., Test of General Relativity and Measurement of the Lense-Thirring Effect with Two Earth Satellites, Science, 279, 2100-2103, 1998.

Ciufolini, I., Currie, D., Iorio, L., Paolozzi, A., Lucchesi, D., Pavlis, E.C., Peron, R., Rubincam, D., and Smith, B.R., INFN Study on WEBER-SAT/LARES, Università di Lecce, 2004.

Ciufolini, I., and Pavlis, E.C., A confirmation of the general relativistic prediction of the Lense-Thirring effect, Nature, 431, 958960, 2004.

Everitt, F., et al., Gravity Probe B: Countdown to Launch, in: Lämmerzahl, C., Everitt, C.W. F., and Hehl, F.W. (ed.), Gyros, Clocks, Interferometers...: Testing Relativistic Gravity in Space, pp. 52-82, Springer, Berlin, 2001.

Iorio, L., Earth tides and Lense-Thirring effect, Celest. Mech. \& Dyn. Astron., 79, 201-230, 2001.

Iorio, L., A critical approach to the concept of a polar, low-altitude LARES satellite, Class. Quantum Grav., 19, L175-L183, 2002a.

Iorio, L., Is it possible to improve the present LAGEOS-LAGEOS II Lense-Thirring experiment?, Class. Quantum Grav., 19, 54735480, 2002b.

Iorio, L., A reassessment of the systematic gravitational error in the LARES mission, Gen. Rel. Grav., 35, 1263-1272, 2003a.

Iorio, L., A new proposal for measuring the Lense-Thirring effect with a pair of supplementary satellites in the gravitational field of the Earth, Phys. Lett. A, 308, 81-84, 2003b.

Iorio, L., On a new observable for measuring the Lense-Thirring effect with Satellite Laser Ranging, Gen. Rel. Grav., 35, 1583-1595, 2003c. 
Iorio, L., The impact of the static part of the Earth's gravity field on some tests of General Relativity with Satellite Laser Ranging, Celest. Mech. and Dyn. Astron., 86, 277-294, 2003d.

Iorio, L., Recent Developments in Testing General Relativity with Space Geodetic Techniques, in: Lynch, T.V. (ed.), Horizons in World Physics, vol. 245, pp.1-25, Nova Science Publishers, Hauppauge, 2004.

Iorio, L., On the reliability of the so far performed tests for measuring the Lense-Thirring effect with the LAGEOS satellites, New Astronomy, in press, 2005a.

Iorio, L., On the impossibility of using the longitude of the ascending node of GP-B for measuring the Lense-Thirring effect, Gen. Rel. Grav., 37, 1-8, 2005b.

Iorio, L., and D.M. Lucchesi, LAGEOS-type Satellites in Critical Supplementary Orbital Configuration and the Lense-Thirring Effect Detection, Class. Quantum Grav., 20, 2477-2490, 2003.

Iorio, L., and Morea, A., The impact of the new Earth gravity models on the measurement of the Lense-Thirring effect, Gen. Rel. Grav., 36, 1321-1333, 2004.

Iorio, L., and Doornbos, E., On the possibility of using Jason-1 in determining the Lense-Thirring effect, preprint http://www.arxiv.org/abs/gr-qc/0404062, Gen. Rel. Grav., in press, 2005.

Iorio, L., D.M. Lucchesi, and I. Ciufolini, The LARES Mission Revisited: An Alternative Scenario, Class. Quantum Grav., 19, 43114325, 2002.

Iorio, L., Ciufolini, I., Pavlis, E.C., Schiller, S., Dittus, H., and Lämmerzahl, C., On the possibility of measuring the LenseThirring effect with a LAGEOS-LAGEOS II-OPTIS mission, Class. Quantum Grav., 21, 2139-2151, 2004.

Lämmerzahl, C. Ciufolini, I., Dittus, H., Iorio, L., Müller, H., Peters, A., Samain, E., Scheithauer, S., and Schiller, S., OPTIS-An Einstein Mission for Improved Tests of Special and General Relativity, Gen. Rel. Grav., 36, 2373-2417 2004. 
Lemoine, F. G., Kenyon, S. C., Factor, J. K., Trimmer, R. G., Pavlis, N. K., Chinn, D. S., Cox, C. M., Klosko, S. M., Luthcke, S. B., Torrence, M. H., Wang, Y. M., Williamson, R. G., Pavlis, E. C., Rapp, R. H., and Olson, T. R. (1998). The Development of the Joint NASA GSFC and the National Imagery Mapping Agency (NIMA) Geopotential Model EGM96 NASA/TP-1998-206861.

Lense, J., and Thirring, H., Über den Einfluss der Eigenrotation der Zentralkörper auf die Bewegung der Planeten und Monde nach der Einsteinschen Gravitationstheorie, Phys. Z., 19, 156-163, 1918, translated by Mashhoon, B., Hehl, F.W., and Theiss, D.S., On the Gravitational Effects of Rotating Masses: The Thirring-Lense Papers, Gen. Rel. Grav., 16, 711-750, 1984.

Lucchesi, D., Reassessment of the error modelling of nongravitational perturbations on LAGEOS II and their impact in the Lense-Thirring determination. Part I, Plan. Space Sci., 49, 447463, 2001.

Lucchesi, D., Reassessment of the error modelling of nongravitational perturbations on LAGEOS II and their impact in the Lense-Thirring determination. Part II, Plan. Space Sci., 50, 10671100, 2002.

Lucchesi, D., The asymmetric reflectivity effect on the LAGEOS satellites and the germanium retroreflectors, Geophys. Res. Lett., 30, 1957, 2003.

Lucchesi, D., LAGEOS Satellites Germanium Cube-CornerRetroreflectors and the Asymmetric Reflectivity Effect, Celest. Mech. Dyn. Astron., 88, 269-291, 2004.

Lucchesi, D., Ciufolini, I., Andrés, J.I., Pavlis, E.C., Peron, R., Noomen, R., and Currie, D.G., LAGEOS II perigee rate and eccentricity vector excitations residuals and the Yarkovsky-Schach effect, Plan. Space Sci., 52, 699-710, 2004.

Nordtevdt, K., Gravitomagnetic interaction and laser ranging to Earth satellites , Phys. Rev. Lett., 61, 2647-2649, 1988.

Nordtvedt, K., Some considerations on the varieties of frame dragging, in: Ruffini, R.J., and Sigismondi, C. (ed.), Nonlinear Gravito- 
dynamics. The Lense-Thirring Effect, pp. 35-45, World Scientific, Singapore, 2003.

Peterson, G.E., Estimation of the Lense-Thirring precession using laser-ranged satellites, CSR-9\%-1, Center for Space Research, The University of Texas at Austin, 1997.

Reigber, Ch., Schwintzer, P., Stubenvoll, R., Schmidt, R., Flechtner, F., Meyer, U., König, R., Neumayer, H., Förste, Ch., Barthelmes, F., Zhu, S.Y., Balmino, G., Biancale, R., Lemoine, J.-M., Meixner, H., and Raimondo, J.C., A High Resolution Global Gravity Field Model Combining CHAMP and GRACE Satellite Mission and Surface Gravity Data: EIGEN-CG01C, submitted to J. of Geodesy, 2004.

Reigber, Ch., Schmidt, R., Flechtner, F., König, R., Meyer, U. Neumayer, K.-H., Schwintzer, P. and Zhu, S.Y. An Earth gravity field model complete to degree and order 150 from GRACE: EIGENGRACE02S, J. of Geodyn., 39, 1-10, 2005.

Ries, J.C., Eanes, R.J. and Tapley, B.D., Lense-Thirring Precession Determination from Laser Ranging to Artificial Satellites, in: Ruffini, R.J., and Sigismondi, C. (ed.), Nonlinear Gravitodynamics. The Lense-Thirring Effect, pp. 201-211, World Scientific, Singapore, 2003.

Schiff, L., On Experimental Tests of the General Theory of Relativity, Am. J. Phys., 28, 340-343, 1960.

Stella, L. Cui, W., Chen, W., Zhang, S.N., Van Der Klis, M., Karas, V., Semerák, O., De Felice, F., Dovčiak, M., Casini, H., Montemayor, R., Morsink, S.M., Silbergleit, A.S., Wagoner, R.V., Khanna, R., Markovic, D., and Lamb, F.K., Section E: Probing the Gravitomagnetic Lense-Thirring effect with Neutron Stars and Black Holes, in: Ruffini, R.J., and Sigismondi, C. (ed.), Nonlinear Gravitodynamics. The Lense-Thirring Effect, pp. 235-345, World Scientific, Singapore, 2003.

Tapley, B.D., and Ciufolini, I., Measuring the Lense-Thirring precession using a second LAGEOS satellite, CSR-89-3, Center for Space Research, The University of Texas at Austin, 1989. 
Vespe, F., and Rutigliano, P., The improvement of the Earth gravity field estimation and its benefits in the atmosphere and fundamental physics, paper presented at 35th COSPAR Scientific Assembly Paris, France, 18 - 25 July 2004, COSPAR04-A-03614, submitted to Adv. Sp. Res., 2004. 